\title{
QUANTIFICAÇÃO DA DIVERGÊNCIA GENÉTICA ENTRE ACESSOS DE GOIBEIRA POR MEIO DA ESTRATÉGIA WARD-MLM ${ }^{1}$
}

\author{
BIANCA MACHADO CAMPOS ${ }^{2}$, ALEXANDRE PIO VIANA ${ }^{3}$, SILVANA SILVA RED QUINTAL 4 , \\ LEANDRO SIMÕES AZEREDO GONÇALVES ${ }^{5}$, PATRÍCIA GOMES DE OLIVEIRA PESSANHA ${ }^{6}$
}

RESUMO- O presente trabalho teve como objetivo quantificar a divergência genética entre 138 acessos de goiabeira procedentes do banco de germoplasma da Universidade Estadual do Norte Fluminense Darcy Ribeiro (UENF), com base em descritores morfológicos, agronômicos e físico-químicos, por meio do procedimento Ward - Modified Location Model (MLM). Para tanto, foram avaliados 13 descritores, sendo cinco qualitativos (coloração da polpa, superfície do fruto, formato do fruto ao final do pedúnculo, largura do pescoço e uniformidade da cor da polpa) e oito quantitativos (massa média do fruto, diâmetro longitudinal do fruto, diâmetro transversal do fruto, rendimento da polpa, teor de sólidos solúveis totais, acidez do fruto, relação teor de sólidos solúveis totais e acidez do fruto e teor de ácido ascórbico). Detectou-se ampla variabilidade genética pelos dados morfológicos, agronômicos e físico-químicos nos 138 acessos de goiaba. Pelo procedimento da função da verossimilhança, determinou-se oito o número ideal de grupos, com um valor de incremento de 67,51. O grupo III foi considerado o mais distante, enquanto os grupos I, II, IV, V e VI, os mais próximos. O procedimento Ward-MLM é uma ferramenta útil para detectar divergência genética e agrupar os acessos utilizando, simultaneamente, variáveis qualitativas e quantitativas.

Termos para indexação: Psidium guajava L., variabilidade genética, análise conjunta, caracterização do germoplasma.

\section{QUANTIFICATION OF THE GENETIC DIVERGENCE AMONG GUAVA ACCESSIONS USING WARD-MLM STRATEGY}

\begin{abstract}
The present study aimed at evaluating the genetic divergence among 138 guava accessions coming from the Germplasm Collection of the Universidade Estadual do Norte Fluminense Darcy Ribeiro (UENF) based on morphological, agronomic and physic-chemical traits by Ward - Modified Location Model (MLM). Thirteen descriptors were evaluated, five qualitative (flesh color, fruit surface, shape at stalk end , neck width and uniformity of the flesh color) and eight quantitative (fruit weight, fruit diameter, fruit length, pulp revenue, soluble solids contents, total titratable acidity, total soluble solids/total titratable acidity ratio and ascorbic acid). High genetic variability was detected considering morphological, agronomic and physicchemical traits in the 138 guava accessions studied. The procedure of the likelihood function showed that the ideal numbers of groups was eight, with an increment value of 67.51. Group III was considered the most distant, while groups I, II, IV, V and VI the closest. The Ward- MLM procedure is a useful tool for detecting genetic divergence and cluster accessions using both qualitative and quantitative variables.
\end{abstract}

Index terms: Psidium guajava L., genetic variability, joint analysis, germplasm characterization.

\footnotetext{
${ }^{1}$ (Trabalho 208-12). Recebido em: 05-07-2012. Aceito para publicação em: 15-03-2013.

${ }^{2}$ Eng. Ftal., M.Sc. Campos, RJ, UENF/CCTA/LMGV, CEP 28015620, E-mail: biancamachado@hotmail.com

${ }^{3}$ Eng. Agro, D.Sc., Professor UENF/CCTA/LMGV, CEP 28015620, E-mail: pirapora@uenf.br

${ }^{4}$ Eng. Agro, M. Sc., Campos, RJ, UENF/CCTA/LMGV, CEP 28015620, E-mail: silvanared@hotmail.com

${ }^{5}$ Eng. Agro, D.Sc. UENF/CCTA/LMGV, CEP 28015620, E-mail: 1sagrural@yahoo.com.br

${ }^{6}$ Eng. Agro, D.Sc. UENF/CCTA/LMGV, CEP 28015620, E-mail: patriciagop@uenf.br
} 


\section{INTRODUÇÃO}

A goiabeira (Psidium guajava L., família Myrtaceae) é uma fruteira de elevada importância, distribuída em várias regiões do globo, principalmente na América e na Austrália. Seu fruto é considerado bastante nutritivo e saboroso, sendo caracterizado por um alto conteúdo de pectina, fibra dietética, minerais, aminoácidos essenciais e vitamina C (CORRÊA et al., 2011). Além disso, as diferentes partes da planta (frutos, folhas, raízes e cascas) são amplamente utilizadas na medicina tradicional para tratamentos de diarreia, gastroenterite, disenteria, dores de estômago e indigestão (GUTIÉRREZ et al., 2008; BIRDI et al., 2010; RAI et al., 2010).

No Brasil, a goiaba apresenta lugar de destaque entre as frutas tropicais, em razão principalmente da grande variedade de produtos e subprodutos, usos e forma de consumo.

Entre as principais cultivares disponíveis aos produtores brasileiros, destacam-se as cultivares 'Paluma', (PEREIRA; RYOSUKE, 2011). 'Rica', 'Sassaoka', 'Pedro Sato', 'Cortibel' e 'Século XXI', que foram desenvolvidas a partir de seleções de produtores ou por instituições de pesquisa em pomares de sementes de polinização aberta (POMMER et al., 2006; SANTOS et al., 2011). Logo, a avaliação dessas populações segregantes obtidas de polinização aberta torna-se uma importante estratégia no que diz respeito à obtenção de cultivares e na indicação de combinações parentais que produzam progênies com o máximo de variabilidade genética, aumentando-se, assim, a oportunidade de obtenção de indivíduos superiores.

Nesse contexto, o uso de algoritmos multivariados que vise a quantificar a dissimilaridade entre os indivíduos é considerado uma ferramenta essencial para o melhoramento de plantas (GONÇALVES et al., 2008). Dentre os métodos multivariados, o procedimento Ward - Modified Location Model (MLM), proposto por Franco et al. (1998), consiste em uma excelente estratégia para a quantificação da variabilidade, utilizando as variáveis quantitativas e qualitativas simultaneamente.

Diversos trabalhos vêm sendo desenvolvidos, utilizando tal procedimento, como, por exemplo, com milho (GUTIÉRREZ et al., 2003; FRANCO et al., 2005; ORTIZ et al., 2008), nabo-forrageiro (PADILHA et al., 2005), tomate (GONÇALVES et al., 2009), feijão (BARBÉ et al., 2010; CABRAL et al., 2010), pimenta/pimentão (SUDRÉ et al., 2010) e banana (PESTANA et al., 2011).

Sendo assim, o presente trabalho teve como objetivo quantificar a divergência genética entre
138 acessos de goiabeira com base em descritores morfológicos, agronômicos e físico-químicos, por meio do procedimento Ward-MLM.

\section{MATERIAL E MÉTODOS}

Foram avaliados 138 genótipos de goiabeira obtidos de cruzamentos controlados biparentais de indivíduos oriundos do sítio Providência- Bom Jesus do Itabapoana- RJ, que fazem parte do banco de germoplasma da Universidade Estadual do Norte Fluminense Darcy Ribeiro (UENF).

Os genitores utilizados para cruzamento foram selecionados por análise molecular e estudo da diversidade genética (PESSANHA et al., 2011). As sementes dos frutos desses cruzamentos que vieram de genitores com considerável grau de heterozigoze deram origem a uma população $F_{1}$ de ampla variabilidade genética, devido à população $\mathrm{F}_{1}$ estar comportando-se com $\mathrm{F}_{2}$, ou seja, uma população segregante. Essa população $F_{1}$ se trata dos 138 genótipos avaliados.

O trabalho foi conduzido na área experimental do Colégio Agrícola Antônio Sarlo, em Campos dos Goytacazes, sendo o espaçamento adotado de 1,5 m entre plantas e 3,0 m entre linhas.

Os acessos foram plantados em junho de 2009 , no qual a primeira poda de formação foi realizada no mês de dezembro de 2009 , e a poda de frutificação, no mês de setembro de 2010 . O período de colheita ocorreu nos meses de fevereiro a abril de 2011. Todas as plantas receberam os tratos culturais recomendados para cultura, como a condução da planta, adubação, irrigação, controle das plantas daninhas e controle de pragas e doenças.

No período de frutificação, os frutos com tamanho de aproximadamente $10 \mathrm{~cm}$ foram ensacados com saco de polietileno. Posteriormente, quando os frutos atingiram o estádio 1 de maturação com a cor da casca verde-escura, estes foram colhidos para posterior avaliação no laboratório de Melhoramento Genético Vegetal da UENF. Foram amostrados 10 frutos por planta para o estudo de caracterização e avaliação dos acessos.

Na caracterização e na avaliação dos acessos, foram utilizados 13 descritores, propostos pela International Union for the Protection of New Varieties of Plants (UPOV), sendo cinco qualitativos e oito quantitativos. Os descritores qualitativos foram: coloração da polpa, uniformidade da cor da polpa, superfície do fruto, formato do fruto ao final do pedúnculo e largura do pescoço. Em relação aos descritores quantitativos, foram utilizados: massa média do fruto, diâmetro longitudinal do fruto, diâmetro transversal do fruto, 
rendimento da polpa, acidez do fruto, teor de sólidos solúveis totais, relação do teor de sólidos solúveis e acidez, e o teor de ácido ascórbico.

As variáveis quantitativas e qualitativas foram analisadas simultaneamente, utilizando o procedimento Ward-MLM para composição dos grupos de acessos por meio do procedimento CLUSTER e IML do programa SAS. Para uso do método de agrupamento Ward, a matriz de distância foi obtida pelo algoritmo de Gower (GOWER, 1971). A definição do número ideal de grupos foi realizada de acordo com os critérios do pseudo-F e pseudo- $\mathrm{t}^{2}$, combinado com o perfil da verossimilhança, associado com o teste da razão da verossimilhança. A estratégia Ward- MLM consiste em duas fases, sendo que, na primeira fase, os grupos são definidos pelo método de agrupamento Ward (WARD, 1963), usando a matriz de dissimilaridade de Gower (GOWER, 1971), e na segunda fase, a média do vetor da variável quantitativa para cada subpopulação, independentemente dos valores da variável qualitativa, sendo estimados pelo procedimento MLM.

O gráfico da diferença entre os grupos e a correlação das variáveis com a variável canônica foi estabelecido com o uso do procedimento CANDISC do programa SAS. A distância adaptada por Krzanowski (1983) e posteriormente por Franco et al. (1998), para a distribuição das variáveis conjuntas (quantitativas e qualitativas), foi usada para a determinação da dissimilaridade entre os grupos formados.

\section{RESULTADOS E DISCUSSÃO}

Pela caracterização morfológica dos frutos, pode-se observar, para a coloração da polpa, predominância da cor rosa $(63,67 \%)$, seguida pelas cores rosa-pálida, branca, rosa- escura e rosa-alaranjada com 21,$74 ; 7,24 ; 6,52$ e $0,73 \%$, respectivamente. Para o consumo interno, a preferência é pela goiaba de polpa rosa-escura, enquanto no mercado externo, a goiaba de polpa branca é preferida. Quanto à uniformidade da cor da polpa, 65,94\% contiveram frutos com cor de polpa uniforme, enquanto $34,08 \%$ apresentaram frutos com cor de polpa desuniforme.

Em relação ao pescoço do fruto, $92,03 \%$ dos acessos foram ausentes, enquanto 5,07;2,07 e 0,73\% dos acessos apresentaram frutos com pescoço largo, médio e estreito, respectivamente. Para superfície do fruto, $48,55 \%$ dos acessos apresentaram frutos lisos, $45,65 \%$ frutos ásperos e 5,80\% acidentados. Já quanto ao formato do fruto ao final do pedúnculo, houve predomínio para formato arredondado $(37,68 \%)$, seguido pelos formatos truncado $(26,09 \%)$, pontudo
$(21,04 \%)$, largamente arredondado $(7,97 \%)$ e com pescoço $(7,25 \%)$. Esses descritores mostram-se importantes na escolha de genótipos visando ao consumo in natura, pois estão relacionados a fatores que depreciam o produto, caso estes não sejam atrativos aos consumidores.

Com base nos descritores quantitativos, detectou-se ampla variação fenotípica entre os acessos avaliados. A massa média dos frutos variou de 131,20 a 410,92 g, com média de 235,60 g. Segundo Gonzaga Neto et al. (1987) e Lima et al. (2002), a massa média do fruto é uma característica importante, uma vez que os frutos de maior massa são também os de maior tamanho, e estes, por sua vez, são mais atrativos ao consumidor.

O comprimento e o diâmetro do fruto contiveram uma amplitude de 59 a $82,62 \mathrm{~mm}$ e de 64,30 a 101,39 mm, respectivamente, entre os acessos avaliados. A análise dessas variáveis, isoladamente, tem pouca importância para a caracterização dos frutos dos genótipos de goiabeira (LIMA et al., 2002). Contudo, a relação entre essas variáveis é bastante representativa, pois indica o formato do fruto, e quanto mais próximo de 1 , mais redondo. Nesse sentido, essa relação variou de 0,99 a 1,40 entre os acessos estudados. Para o rendimento da polpa, a média dos acessos avaliados foi de $80,07 \%$, com uma variação de 74,15 a $86,02 \%$, valores acima de $80 \%$ são aceitáveis para a seleção de frutos de goiabeira.

Com relação à composição química, os frutos apresentaram teores de sólidos solúveis (SST) de 6,06 a $11,36{ }^{\circ}$ BRIX e acidez titulável (AT) de 0,224 a $0,646 \mathrm{~g}$ de ácido cítrico x $100 \mathrm{~g}$ de polpa, o que resultou numa relação SST/AT de 11,55 a 33.82. Já o teor de Vitamina C variou de 10,30 a 177,79 mg de ácido ascórbico x $100 \mathrm{~g}$ de polpa. Pereira et al. (2003), avaliando a composição química de três cultivares comerciais ('Rica', 'Paluma' e 'século XXI'), verificaram teores de SST (de 9,4; 9,6 e 9, $8^{\circ}$ BRIX, respectivamente), AT (de 0,53; 0,52 e 0,47 g de ácido cítrico x $100 \mathrm{~g}$ de polpa, respectivamente) e Vitamina C (de 107,0; 57,7 e 84,9 mg de ácido ascórbico x $100 \mathrm{~g}$ de polpa, respectivamente). Contudo, vale ressaltar que os teores de SST, AT e vitamina $\mathrm{C}$ podem variar de acordo com diversos fatores, principalmente com o grau de maturação utilizado durante as análises.

Pelo procedimento da função da verossimilhança, determinou-se oito o número ideal de grupos, com um valor de incremento de 67,51 (Figura 1). Segundo Gonçalves et al. (2009) e Barbé et al. (2010), a análise da função da verossimilhança pode definir critérios mais precisos na formação dos grupos, resultando na determinação de grupos menos 
subjetivos. Padilha et al. (2005), avaliando a diversidade de 120 acessos de Brassica rapa subsp. rapa L., verificaram que o maior incremento na função da probabilidade foi atingido quando cinco grupos foram considerados. Por sua vez, Ortiz et al. (2008), estudando raças de milho de altas altitudes do Peru, observaram que os maiores incrementos na função da probabilidade foram constatados na constituição de quatro e oito grupos.

Os oito grupos formados foram constituídos por $36 ; 17 ; 3 ; 13 ; 25 ; 22 ; 16$ e 6 acessos (Tabela 1 ). Para característica coloração da polpa do fruto, os dez acessos que apresentaram coloração branca ficaram alocados nos grupos I, II, IV, V e VIII, enquanto a coloração rosa foi amplamente distribuída em todos os grupos. Em relação à superfície do fruto, os grupos I e VI contiveram um predomínio de acessos com frutos lisos, enquanto os grupos II, V e VIII constituíram acessos com predomínio de frutos ásperos. Os acessos que apresentaram frutos com protuberâncias foram alocados nos grupos $\mathrm{V}$ (quatro acessos), grupo II (dois acessos) e grupo I e VIII (um acesso para cada um).

Para a característica formato do fruto ao final do pedúnculo, os grupos apresentaram uma ampla distribuição das classes, sendo os grupos I, II e VI com predomínio de frutos arredondados e o grupo $\mathrm{V}$ com predomínio de frutos truncados. Os acessos que contiveram frutos com pescoço foram agrupados nos grupos VI (um acesso), VII (quatro acessos) e IV (cinco acessos). Em relação à uniformidade da cor da polpa, os grupos I, II, III, V, VII e VIII apresentaram predominantemente acessos com coloração de polpa uniforme, enquanto os grupos IV e VI predominaram acessos com coloração de polpa desuniforme.

Com base nos descritores agronômicos quantitativos, o grupo IV conteve os acessos com os menores valores para massa média dos frutos, diâmetro longitudinal do fruto, diâmetro transversal do fruto, rendimento da polpa, teor de sólidos solúveis totais e acidez do fruto (Tabela 2). Já os acessos com os maiores valores para massa média dos frutos foram alocados nos grupos VIII, V e II, com 283,65;269,81 e $261,73 \mathrm{~g}$, respectivamente; enquanto para rendimento de polpa, os maiores valores foram reunidos nos grupos VIII, VI e V, com 83,52; 82,21 e 80,61\%, respectivamente.

Em relação à composição química dos frutos, o grupo VIII reuniu os acessos com os maiores valores para SST, com $9,42^{\circ}$ Brix; seguido pelos grupos VI e VII com 8,91 e 8,25, respectivamente (Tabela 2). Para AT, os acessos com os menores valores foram alocados nos grupos IV e VII (0,26 e 0,27 g de ácido cítrico x $100 \mathrm{~g}$ de polpa, respectivamente); enquan- to o grupo III obteve os acessos com os maiores valores, com uma média de $0,55 \mathrm{~g}$ de ácido cítrico x $100 \mathrm{~g}$ de polpa. Já para vitamina C, os grupos III e VIII reuniram os acessos com os maiores teores, com 81,69 e 75,55 mg de ácido ascórbico x 100g de polpa, respectivamente.

$\mathrm{Na}$ análise baseada nas variáveis canônicas (VC), foi verificado que as duas primeiras variáveis foram responsáveis por apenas $61,79 \%$, sendo $\mathrm{VC} 1$ responsável por 39,12\% e VC2 por 22,68\%. Logo, um gráfico bidimensional não capitalizaria uma boa proporção da variabilidade total, sendo necessária a introdução da terceira variável, que foi responsável por 19,50\%. Logo, a representação tridimensional é a mais adequada para a representação do conjunto de dados, sendo o somatório das três variáveis responsável por $81,30 \%$ da variação total (Figura 2). Gonçalves et al. (2009), trabalhando com tomate, verificaram que as duas primeiras variáveis canônicas explicaram cerca de $90 \%$ da variabilidade entre os grupos, e o gráfico bidimensional mostrou-se adequado para a visualização da relação entre os grupos. As maiores correlações das variáveis com a primeira variável canônica foram o rendimento de polpa e a relação SST/AT;com a segunda variável canônica foram AT e SST/AT, e com a terceira variável canônica foram rendimento de polpa e comprimento do fruto (Tabela 1).

Pela análise gráfica das VCs, percebe-se um distanciamento do grupo III dos demais grupos formados pelo procedimento Ward-MLM e a aproximação dos grupos I, II, IV, V, VI e VIII (Figura 2). Esses resultados estão em consonância aos obtidos pela distância proposta por Franco et al. (1998), no qual se verifica que os grupos III e VI são os mais distantes $(35,67)$, enquanto os grupos VI e VIII são os mais próximos $(5,31)$ (Tabela 3$)$. Percebe-se que o grupo III apresentou melhor desempenho para variáveis AT e Vit. C, e o grupo VIII, para variáveis MMF, DF, CF, RP, SST e SST/AT. Esses grupos reuniram acessos com melhores desempenhos agronômicos e físico-químicos, apresentando um distanciamento de 28,63 e, portanto, podem ser um indicativo de possíveis cruzamentos de acessos entre os dois grupos, visando à complementação de alelos favoráveis para as características avaliadas, já que, de acordo com essas características, esses grupo seriam complementares. 
TABELA 1- Variáveis e número de acessos por grupo de características qualitativas em cada um dos oito grupos (G1, G2, G3, G4, G5, G6, G7 e G8), formados pela estratégia Ward-MLM.

\begin{tabular}{|c|c|c|c|c|c|c|c|c|}
\hline \multirow{2}{*}{ Variáveis } & \multicolumn{8}{|c|}{ Grupos } \\
\hline & G1(36) & G2 (17) & G3 (3) & G4 (13) & G5 (25) & G6 (22) & G7 (16) & G8 (6) \\
\hline \multicolumn{9}{|c|}{ Coloração da polpa do fruto } \\
\hline Branco & 2 & 1 & - & 1 & 5 & - & - & 1 \\
\hline Rosa-pálido & 7 & 3 & 1 & 3 & 10 & 2 & 3 & 1 \\
\hline Rosa & 24 & 13 & 2 & 8 & 10 & 19 & 11 & 1 \\
\hline Rosa - escuro & 3 & - & - & 1 & - & 1 & 2 & 2 \\
\hline Rosa - Alaranjado & - & - & - & - & - & - & - & 1 \\
\hline \multicolumn{9}{|l|}{ Superfície do fruto } \\
\hline Lisa & 26 & 3 & 1 & 6 & 7 & 16 & 9 & 1 \\
\hline Áspera & 9 & 12 & 2 & 7 & 14 & 6 & 7 & 4 \\
\hline Acidentado & 1 & 2 & - & - & 4 & - & - & 1 \\
\hline \multicolumn{9}{|c|}{ Formato do fruto ao final do pedúnculo } \\
\hline Largamente arredondado & 3 & 4 & 2 & - & 1 & 1 & 1 & - \\
\hline Arredondado & 19 & 9 & 1 & - & 5 & 11 & 5 & 1 \\
\hline Truncado & 6 & 1 & - & 5 & 19 & 1 & 3 & 1 \\
\hline Pontudo & 8 & 3 & - & 3 & - & 8 & 3 & 4 \\
\hline Com pescoço & - & - & - & 5 & - & 1 & 4 & - \\
\hline \multicolumn{9}{|c|}{ Largura do pescoço (quando presente) } \\
\hline Estreito & - & - & - & - & - & - & 1 & - \\
\hline Médio & - & - & - & 2 & - & - & 1 & - \\
\hline Largo & - & - & - & 3 & - & 1 & 2 & - \\
\hline \multicolumn{9}{|c|}{ Uniformidade da cor da polpa } \\
\hline Uniforme & 31 & 13 & 3 & 4 & 18 & 8 & 11 & 4 \\
\hline Desuniforme & 5 & 4 & - & 9 & 7 & 14 & 5 & 2 \\
\hline
\end{tabular}

TABELA 2 - Médias das variáveis quantitativas para cada um dos oito grupos e coeficiente de correlação das variáveis quantitativas com as três primeiras variáveis cananônicas.

\begin{tabular}{|c|c|c|c|c|c|c|c|c|c|c|c|}
\hline \multirow[b]{2}{*}{ Variáveis ${ }^{1}$} & \multicolumn{8}{|c|}{ Grupos } & \multicolumn{3}{|c|}{ CAN } \\
\hline & $\overline{\text { G1(36) }}$ & G2(17) & G3(3) & G4(13) & G5(25) & G6(22) & G7(16) & G8(6) & CAN1 & CAN2 & CAN3 \\
\hline MMF & 211,39 & 261,73 & 250,58 & 159,62 & 269,81 & 213,82 & 226,08 & 283,65 & 0,26 & 0,27 & 0,42 \\
\hline DF & 70,78 & 77,67 & 74,52 & 47,65 & 76,07 & 70,07 & 71,05 & 77,85 & 0,31 & 0,35 & 0,29 \\
\hline $\mathrm{CF}$ & 8 & 83,70 & 83,27 & 54,29 & 89,78 & 79 & 8 & 87 & 0,18 & 0,07 & 0,53 \\
\hline RF & 7,66 & 80,51 & 78,5 & 50,31 & 80,61 & 82,21 & 79 & 83 & 0,48 & 0,02 & 0,64 \\
\hline SST & 7 & 7 & 7,47 & 5,18 & 8,09 & 8 & 8, & 9, & 0,23 & $-0,15$ & 0,37 \\
\hline $\mathrm{A}^{\top}$ & 34 & 0,38 &, 55 & 0,26 & 0,36 & 0,3 & 0,27 & 0,3 & 0,34 & 0,67 & $-0,06$ \\
\hline SST/A & 22,68 & 20,82 & 13,86 & 14,64 & 22,51 & 23,34 & 30,01 & 24, & 0,38 & $-0,64$ & 0,41 \\
\hline Vit C & 43,57 & 43,54 & 81,69 & 35,86 & 44,40 & 34,48 & 27,71 & 75,55 & 0,06 & 0,30 & 0,09 \\
\hline
\end{tabular}

${ }^{1 / M M F}$ : massa média do fruto; DF: diâmetro longitudinal do fruto; CF: diâmetro transversal do fruto; RP: rendimento da polpa; SST: teor de sólidos solúveis totais; AT: acidez do fruto; SST/AT: relação do teor de sólidos solúveis e acidez; e Vit C: teor de ácido ascórbico. 
TABELA 3 - Distância entre os grupos formados pelo procedimento Ward-MLM, proposto por Franco et al. (1998).

\begin{tabular}{cccccccc}
\hline Grupos & I & II & III & IV & V & VI & VII \\
\hline II & 15.06 & & & & & & \\
III & 30.16 & 25.31 & & & & & \\
IV & 14.23 & 14.06 & 27.82 & & & & \\
V & 19.45 & 8.02 & 30.88 & 6.61 & & & \\
VI & 17.72 & 8.26 & 35.67 & 10.18 & 10.46 & & \\
VII & 29.03 & 28.87 & 23.54 & 25.48 & 30.14 & 33.46 & \\
VIII & 19.03 & 6.06 & 28.63 & 11.50 & 5.81 & 5.31 & 23.16 \\
\hline
\end{tabular}

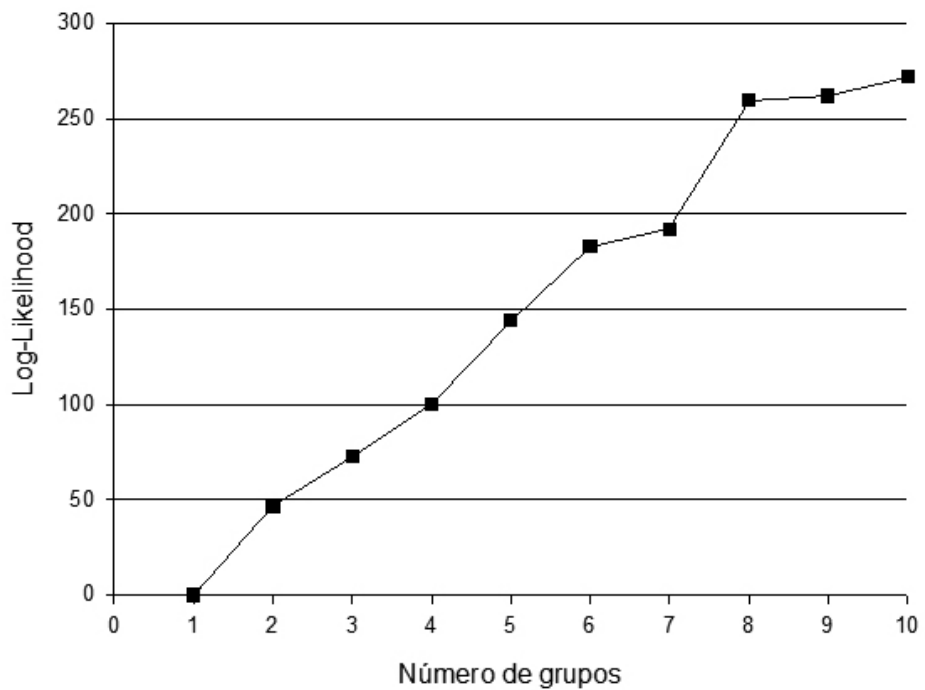

FIGURA 1- Gráfico da função logarítmica da probabilidade (Log-likelihood) em relação ao número de grupos.

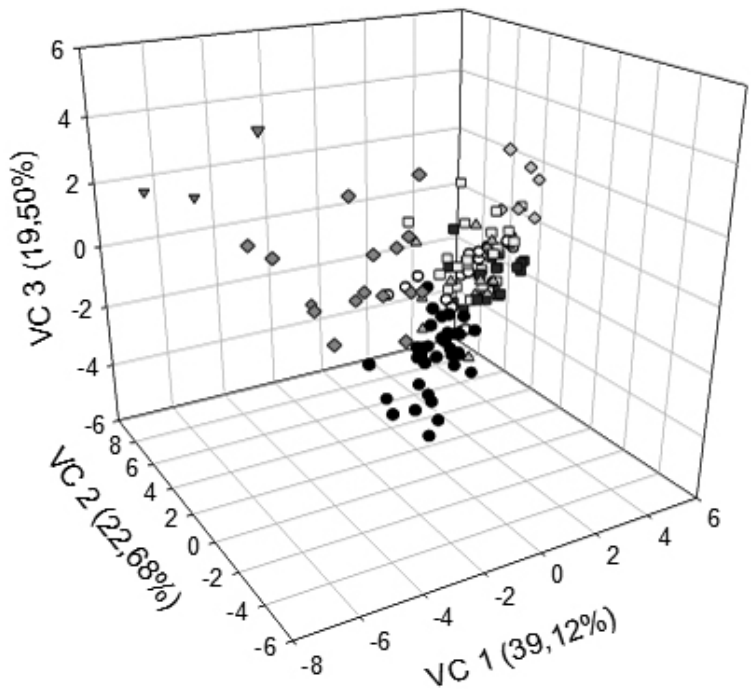

$\begin{array}{ll}\bullet & \text { Grupo I } \\ \bullet & \text { Grupo II } \\ \nabla & \text { Grupo III } \\ \Delta & \text { Grupo IV } \\ \square & \text { Grupo V } \\ \square & \text { Grupo VI } \\ \diamond & \text { Grupo VII } \\ \diamond & \text { Grupo VIII }\end{array}$

FIGURA 2 - Gráfico das três primeiras variáveis canônicas para os oito grupos formados pela análise Ward-MLM. 


\section{CONCLUSÕES}

1- Há variabilidade genética nos 138 acessos de goiaba estudados, considerando-se as variáveis morfológicas, agronômicas e físico-químicas dos frutos.

2- O procedimento estatístico Ward-MLM é uma ferramenta útil para detectar divergência genética e agrupar os acessos, utilizando simultaneamente variáveis quantitativas e qualitativas.

\section{REFERÊNCIAS}

BARBÉ, T. da C.; AMARAL JÚNIOR, A.T. do; GONÇALVES, L.S.A.; RODRIGUES, R.; SCAPIM, C.A. Association between advanced generations and genealogy in inbred lines of snap bean by the Ward-Modified Location Model. Euphytica, Wageningen, v.173, n.3, p.337-343, 2010.

BIRDI, T.; DASWANI, P.; BRIJESH, S.; TETALI, P.; NATU, A.; ANTIA. N. Newer in sights into the mechanism of action of Psidium guajava L. leaves in infectious diarrhoea. BMC Complementary and Alternative Medicine, United Kingdom, v.10, n.1, p. 1-11, 2010 .

CABRAL, P.D.S.; SOARES, T.C.B.; GONÇALVES, L.S.A.; AMARAL JÚNIOR, A.T.; LIMA, A.B.P.; RODRIGUES, R.; MATTA, F.P. Quantification of the diversity among common bean accessions using Ward-MLM strategy. Pesquisa Agropecuária Brasileira, Brasília, v.45, n.10, p.1124-1132, 2010.

CORRÊA, L.C.; SANTOS, C.A.F.; LIMA, G.P.P.; RODRIGUES, M.A.; COSTA, T.P.P. Similaridade genética entre acessos de goiabeiras e araçazeiros baseada em marcadores moleculares AFLP. Revista Brasileira de Fruticultura, Jaboticabal, v. 33, n. 3, p. 859-867, 2011.

FRANCO, J.; CROSSA, J.; TABA, S.; SHANDS, H. A sampling strategy for conserving genetic diversity when forming core subsets. Crop Science, Madison, v.45,n.3, p.1035-1044, 2005.

FRANCO, J.; CROSSA, J.; VILLASEÑOR, J.; TABA, S.; EBERHART, S.A. Classifying genetic resources by categorical and continuous variables. Crop Science, Madison, v.38, n.6, p.1688-1696, 1998.
GONÇALVES, L.S.A.; RODRIGUES, R.; AMARAL JÚNIOR, A.T.; KARASAWA, M.; SUDRÉ, C.P. Comparison of multivariate statistical algorithms to cluster tomato heirloom accessions. Genetics and Molecular Research, Ribeirão Preto, v.7, n.4, p.1289-1297, 2008.

GONÇALVES, L.S.A.; RODRIGUES, R.;AMARAL JÚNIOR, A.T.; KARASAWA, M.; SUDRÉ, C.P. Heirloom tomato gene bank: assessing genetic divergence based on morphological, agronomic and molecular data using Ward-modified location model. Genetics and Molecular Research, Ribeirão Preto, v.8, n.1, p.364-374, 2009.

GONZAGA NETO, A, L.; BEZERRA, J.E.F.; PEDROSA, A.C.; SILVA, H.M. Seleção de cultivares de goiabeira (Psidium guajava L.) para consumo ao natural, na Região do Vale do Rio Moxotó, em Ibibmirim-Pernambuco. Revista Brasileira de Fruticultura, Jaboticabal, v.9, n.2, p.63-66, 1987.

GOWER, J.C. A general coefficient of similarity and some of its properties. Biometrics, Washington, v. 27, p. 857- 874, 1971.

GUTIÉRREZ, L.; FRANCO, J.; CROSSA, J.; ABADIE, T. Comparing a preliminary racial classification with a numerical classification of the maize landraces of Uruguay. Crop Science, Madison, v.43, n.2, p.718-727, 2003.

GUTÍERREZ, R.M.P.; MITCHELL, S.; SOLIS, R.V. Psidium guajava: Are review of its traditional uses phytochemistry and pharmacology. Journal of Ethnopharmacology, Lausanne, v.117, n.1, p. 1-27, 2008.

KRZANOWSKI, W.J. Distance between populations using mixed continuous and categorical variables. Biometrika, London, v.70, n.1, p.235-243, 1983.

LIMA, M.A.C.; ASSIS, J.S.; NETO, L.G. Caracterização dos frutos de goiabeira e seleção de cultivares na região do Submédio São Francisco. Revista Brasileira de Fruticultura, Jaboticabal, v.24, n.2, 2002.

ORTIZ, R.; CROSSA, J.; FRANCO, J.; SEVILLA, R.; BURGUEÑO, J. Classification of Peruvian highland maize races using plant traits. Genetic Resources and Crop Evolution, Drodrechtm, v.55, n.1, p.151-162, 2008. 
PADILLA, G.; CARTEA, M.E.; RODRÍGUEZ, V.M.; ORDÁS, A. Genetic diversity in a germplasm collection of Brassica rapa subsp. rapa L. from northwestern Spain. Euphytica, Wageningen, v.145, n.2, p.171-180, 2005.

PEREIRA, F.M.; CARVALHO, C.A.; NACHTIGAL, J.C. Século XXI: nova cultivar de goiabeira de dupla finalidade. Revista Brasileira de Fruticultura, Jaboticabal, v.25, n.3, p.498-500, 2003.

PEREIRA, F.M.; RYOSUKE, K. Contribuição da pesquisa científica brasileira no desenvolvimento de algumas frutíferas de clima subtropical. Revista Brasileira de Fruticultura, Jaboticabal. v. 33, n. 1- Edição especial. p. 92-108, 2011.

PESTANANA, R.K.N.; AMORIM, E.P.; FERREIRA, C.F.; AMORIM, V.B.O.; OLIVEIRA, L.S.; LEDO, C.A.S.; SILVA, S.O. Agronomic and molecular characterization of gamma ray induced banana (Musa sp.) mutants using a multivariate statistical algorithm. Euphytica, Wageninge, v.178, n.2, p. 151-158, 2011.

PESSANHA, P.G. de O.; VIANA, A.AP.; AMARAL JÚNIOR, A.T.; SOUZA, R.M. de.; TEXEIRA, M. C.; PEREIRA, M. G. Avaliação da diversidade genética em acessos de Psidium ssp. via marcadores RAPD. Revista Brasileira de Fruticultura, Jaboticabal, v.33, n.1, p.129-136. 2011.
POMMER, C.V.; MURAKAMI, K.R.N.; WATLINGTON, F. Goiaba no mundo. O Agronômico, Campinas, v.58, n.1, p. 22-26, 2006.

RAI, M.K.; ASTHANA, P.; JAISWAL, V.S.; JAISWAL U. Biotechnological advances in guava (Psidium guajava L.): recent developments and prospects for further research. Trees, Berlin, v.24, n.1, p.1-12, 2010 .

SANTOS, C.A.F.; CORRÊA, L.C.; COSTA, S.R. Genetic divergence among Psidium accessions based on biochemical and agronomic variables. Crop Breeding and Applied Biotechnology, Londrina, v.11, n.2, p.149-156, 2011.

SUDRÉ, C.P.; GONÇALVES, L.S.A.; RODRIGUES, R.; AMARAL JÚNIOR, A.T. do; RIVA-SOUZA, E.M.; BENTO, C.S. Genetic variability in domesticated Capsicum spp. as assessed by morphological and agronomic data in mixed statistical analysis. Genetics and Molecular Research, Ribeirão Preto, v.9, n.1, p.283-294, 2010.

WARD JUNIOR, J.H. Hierarchical grouping to optimize an objective function. Journal of the American Statistical Association, New York, v.58, n.301, p.236-244, 1963. 